\title{
Buckling and Postbuckling of Unitized, Stiffened Composite Panels
}

\author{
Cyrus J. R. Kosztowny ${ }^{1}$ and Anthony M. Waas ${ }^{2}$ \\ University of Michigan, Ann Arbor, MI, 48109
}

\begin{abstract}
This investigation continues the examination into buckling and postbuckling responses of unitized, stiffened textile fiber reinforced composite plates using the finite element method for comparison with experimental results. Experiments in axial compression of two types of two dimensional, tri-axially braided (2DTBC) textiles are discussed, and a method to recover partially faulty datasets is presented. Comparison of experiments to a developed computational model within the commercial code Abaqus is also discussed. The observed failure mode was successfully changed with the unitized stiffener concept from stiffener debonding to another mode, thereby increasing loading capability well into the postbuckling regime.
\end{abstract}

\section{Introduction}

STIFFENER delamination is a commonly observed catastrophic failure mode for fiber reinforced composite thin$N_{\text {walled structures in axial compression beyond the buckling limit. Such structures are actively used in aerospace }}$ applications because they are lightweight yet exhibit adequate in-plane performance ${ }^{1}$. When the stiffener delaminates and becomes separated from the face sheet or skin, there is a significant reduction in the structure's load carrying capability ${ }^{2}$. Such delamination often leads to an ultimate failure limit load for the structures and is under much study in recent years. Previous research such as stitching has been done that demonstrates a reduced tendency for stiffener delamination ${ }^{3}$. Recently, a new approach was published that takes advantage of new manufacturing concepts with textiles to make stiffened panels where the stiffener is an integral part of the face sheet ${ }^{4}$. There are no extra interfaces generated through this manufacturing technique beyond the interfaces resulting from standard lamination practices. It was proposed in Ref. 4 that the energy needed to delaminate the stiffeners is greatly increased due to the stiffener integration into the face sheet material. Any alternate failure modes that arise due to the inability to delaminate would also have increased energy beyond that needed to delaminate traditionally bonded stiffeners ${ }^{5,6}$.

\section{Experimental System}

\section{A. Specimens}

All manufactured 2DTBC specimens used throughout this investigation are the same as those described in Ref. 4. Dry tri-axially braided textile was infused with an Epon 862 epoxy and EpiKure 3234 hardener system using a vacuum assisted resin transfer molding (VARTM) process. In addition to the axial tows, there are two bias tows braided at $\mathrm{a} \pm \theta^{\circ}$ angle. For the specimens used in this investigation, $\mathrm{a} \pm 30^{\circ}$ and $\mathrm{a} \pm 60^{\circ}$ bias angle was used. Figure 1 provides an example of the $\pm 60^{\circ}$ bias triaxial braid textile and a diagram of the braid for a generic $\pm \theta^{\circ}$ bias tow angle.

Two J-shaped stiffeners were manufactured into the flat plate. A total of four textile layers were used in the stiffened panels, and all layers were of the $\pm 30^{\circ}$ or the $\pm 60^{\circ}$ bias angle. No specimens were a mix of the $\pm 30^{\circ}$ and the $\pm 60^{\circ}$ braided textiles. Other manufacturing details may be found in Ref. 4. Post-manufacturing, the nominal dimensions of the panels were approximately 267 millimeters long by 280 millimeters wide. Each stiffener has a nominal dimension of a 40 millimeter tall web and a 22 millimeter wide flange that forms the J-shape profile. The

${ }^{1}$ NASA Space Technology Research Fellow, Aerospace Engineering, 1320 Beal Avenue, Ann Arbor, MI 48109, AIAA Student Member.

${ }^{2}$ Felix Pawlowski Collegiate Professor of Aerospace Engineering, Aerospace Engineering, 1320 Beal Avenue, Ann Arbor, MI, 48109, AIAA Fellow. 
nominal thickness of each panel is approximately 2.7 millimeters. Due to the manufacturing method and the architecture of the tri-axial braid, however, the thickness varies in a pattern similar to the undulations of the axial and bias tows from the braiding process. The characterization of the nonuniform thickness on the response of the panels is under current investigation. The ends of the stiffened edges were potted in steel putty to act as effectively clamped end boundary conditions ${ }^{7}$. Figure 2 shows the nominal 2DTBC stiffened panels as modeled in Abaqus. Note that the length has been shortened from $267 \mathrm{~mm}$ to $230 \mathrm{~mm}$ in the computational model due to the potted ends. A total of four specimens were manufactured using the $30^{\circ}$ bias angle textile and four specimens were manufactured using the $60^{\circ}$ bias angle textile.
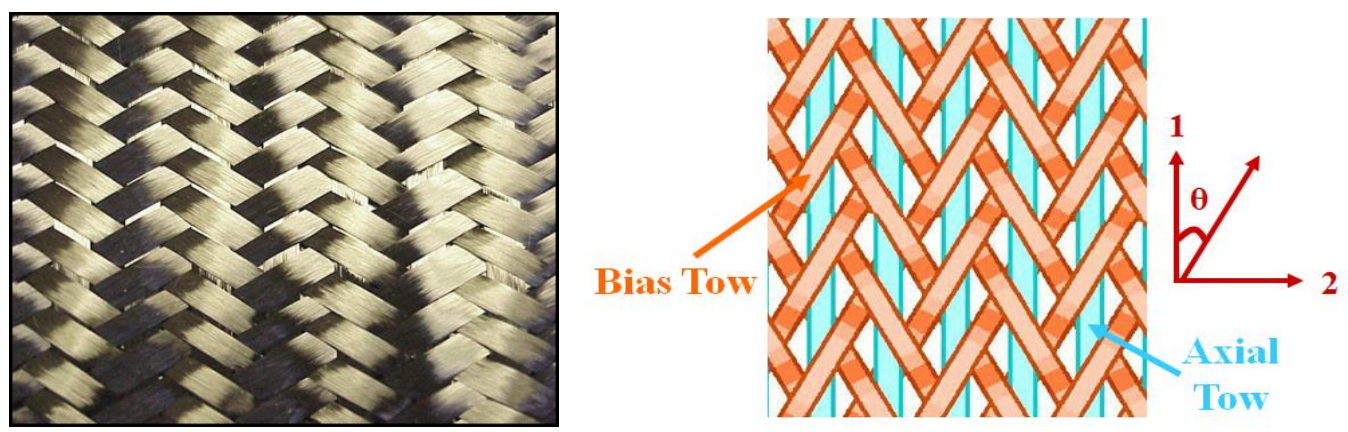

Figure 1. Photo of $\pm 60^{\circ}$ TBC textile (left) and a diagram of the braided axial and bias tow architecture (right).

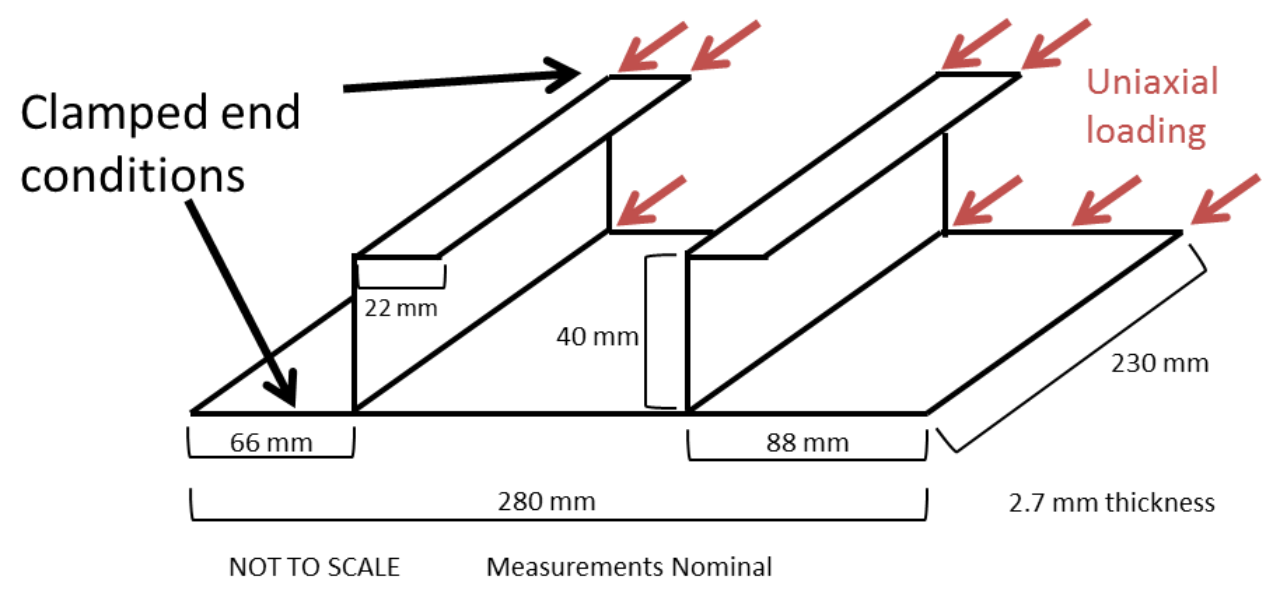

Figure 2. Diagram of nominal geometry for a unitized, stiffened TBC panel as modeled in Abaqus.

\section{B. Experimental Setup in Compression}

A four-post 120-kip Southwark Emery compression loadframe was used to test the initial set of panels (two of the $30^{\circ}$ type and one of the $60^{\circ}$ type) that were presented in Ref. 4 . Due to a fault with the lower platen, however, the instrumented direct current differential transformers (DCDTs) used to measure platen displacement were not able to correctly capture the end-shortening of the specimen. This fault was observed during the initial tests but identified at a later time. A different loadframe was used to test the remaining five specimens. The experimental setup between the two rounds of testing was similar and contained redundant data collection systems.

Four DCDTs were placed between top and bottom platens to record the end-shortening of the specimens throughout each test. 20 strain gages were bonded to the specimens in locations provided in Ref. 4, though the focus of this investigation is on the load and displacement measurements. Each specimen was painted with a speckle pattern for use with optical measurement methods. Two 3D digital image correlation (DIC) systems were used, one on the stiffened side and one on the unstiffened side, to record optical data such as out-of-plane displacements, local end-shortening behavior, and full field strains throughout the test. The DIC systems were redundant with much of 
the physical instrumentation but proved necessary to recover the initial tests' faulty data from the DCDTs. This redundancy allowed all four tests of the $30^{\circ}$ and the $60^{\circ}$ panels to be used for future comparisons with the developed computational model.

\section{First Round of Tests with Suspicious Displacement Recordings}

Figure 3 shows an example of an initial $30^{\circ}$ panel test and how the DCDT load-displacement data were suspicious and severely deviated from the DIC equivalent load-displacement curves. This behavior was observed during the first test, and a second test was conducted because the boundary conditions were reinforced and thought to be inadequate at the time. The enhanced boundary conditions in the second test did not alter the behavior of the recordings and the data were remarkably similar to the test shown in Fig. 3.

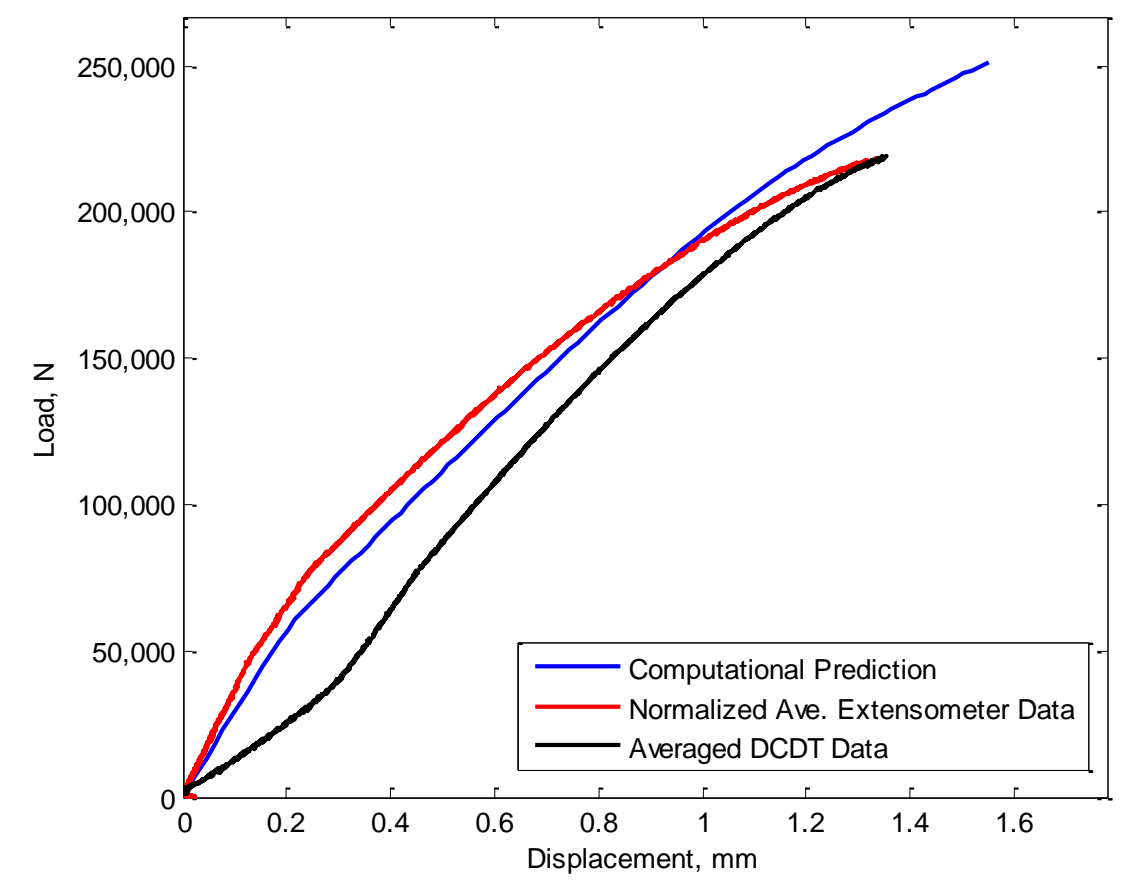

Figure 3. Load-Displacement Curve of TBC 30 P4. Note the significant disagreement between the DCDT and DIC calculated extensometer data in the black and red curves, respectively. This behavior was present for another tested $30^{\circ}$ panel as well as for a $60^{\circ}$ panel - though at a reduced severity in the $60^{\circ}$ panel case.

\section{Second Round of Tests without Suspicious Displacement Recordings}

Figure 4 shows an example of a $30^{\circ}$ panel test conducted on a different loadframe and how the DCDT loaddisplacement data agree much better with the redundant DIC calculated equivalent extensometer data. Also note the excellent agreement between the DCDT data and the computational prediction until approximately $0.21 \mathrm{~mm}$ displacement. The computational prediction will be discussed, but the agreement with the physical DCDTs and the model indicates the underlying behavior was not inherent to the panels but rather the loadframe used in the initial round of tests. 


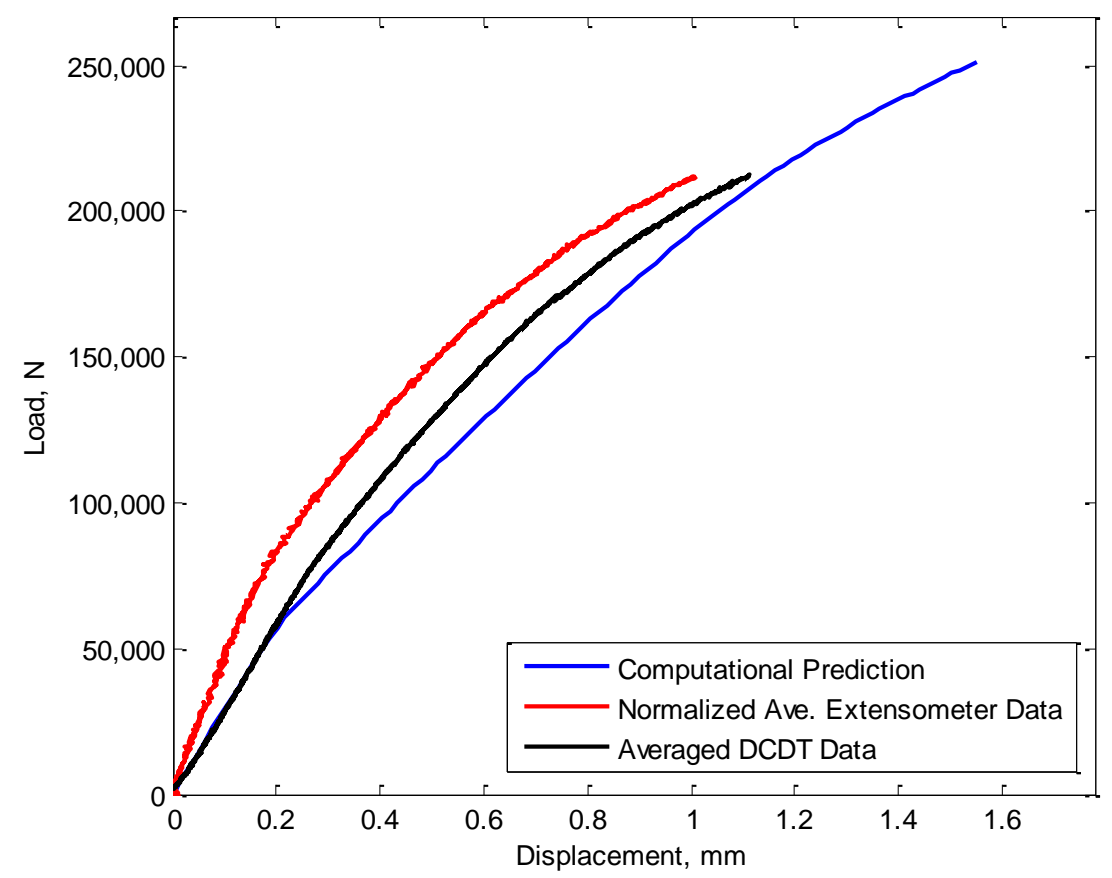

Figure 4. Load-Displacement Curve of TBC 30 P1. Note the increased agreement between the DCDT and DIC calculated extensometer data in the black and red curves, respectively.

\section{Data Recovery}

The recovery of the faulty DCDT data from the initial round of testing was preferred over manufacturing and testing more panels to complete a dataset of four panels for each bias tow braid angle. The redundant DIC data proved key in the recovery of the initial tests because the cameras were recording data independently of the loadframe whereas the DCDTs were mounted on the loadframe. Using the relationship between the DCDT data and the DIC extensometer data in each test, a correlation could be posed on the initial round of data since the panel behavior between the initial and second round tests were observed to be similar.

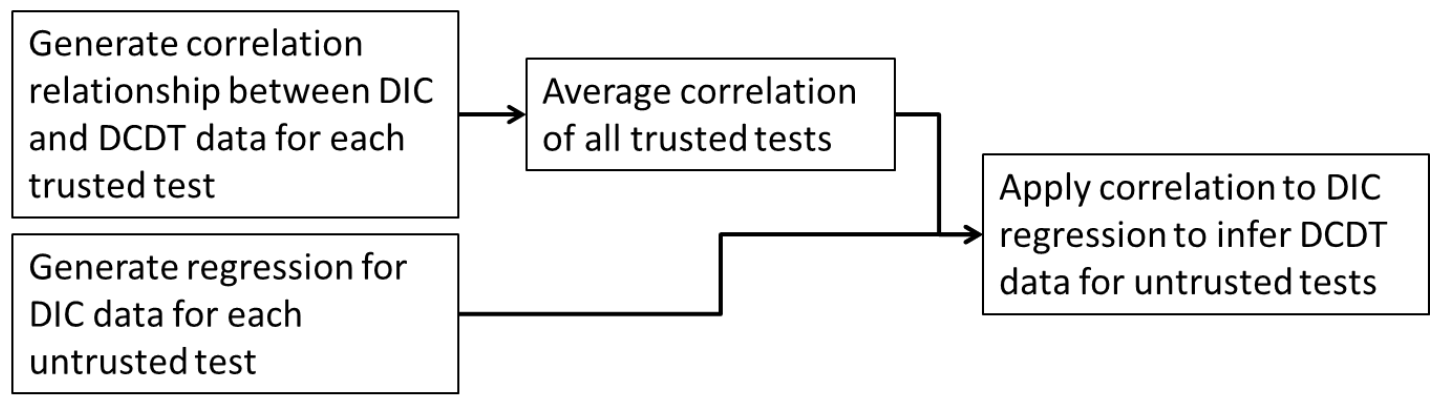

Figure 5. Flow chart demonstrating data recovery process for $30^{\circ}$ and $60^{\circ}$ panels.

Figures 6 and 7 show the correlation between the DIC and DCDT data for the $30^{\circ}$ and $60^{\circ}$ panels, respectively. It is clear that TBC $30 \mathrm{P} 2$ and P4, which were the initial tests, behave differently between the second round TBC $30 \mathrm{P} 1$ and P3 tests. Similarly, TBC 60 P4 was part of the initial round and behaves differently compared to TBC 60 P1-P3 which were part of the second testing round. 


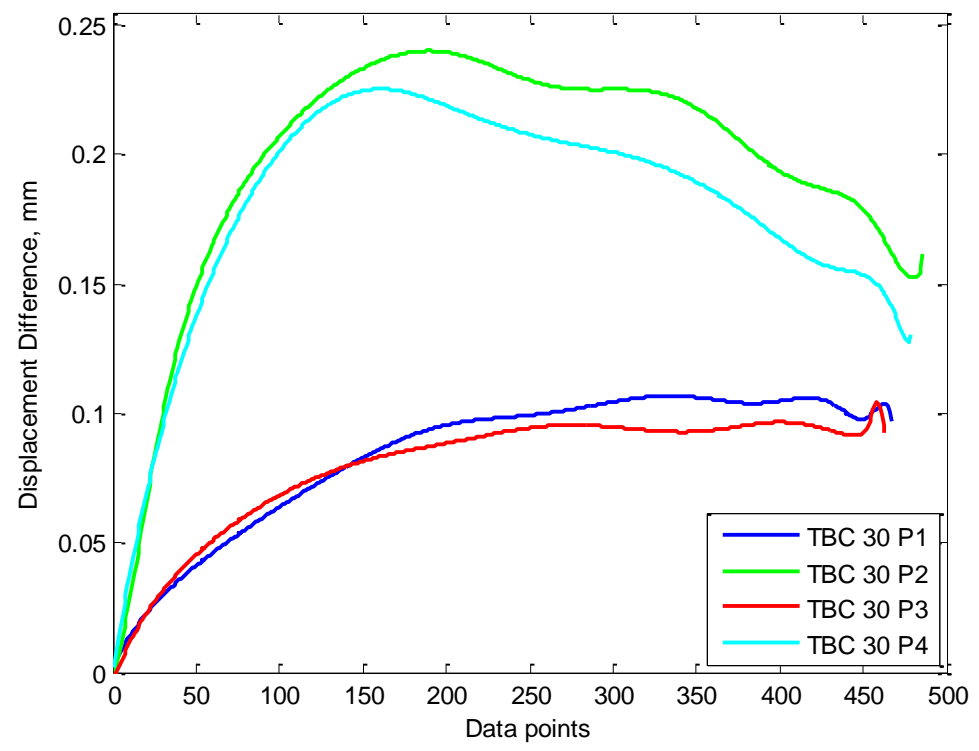

Figure 6. Calculated Displacement Difference for all $\mathbf{T B C ~} 30^{\circ}$ tests. The displacement difference parameter is taken to be the DIC displacement subtracted from the DCDT displacement at a given load. Note the behavior differences between P1 \& P3 and P2 \& P4.

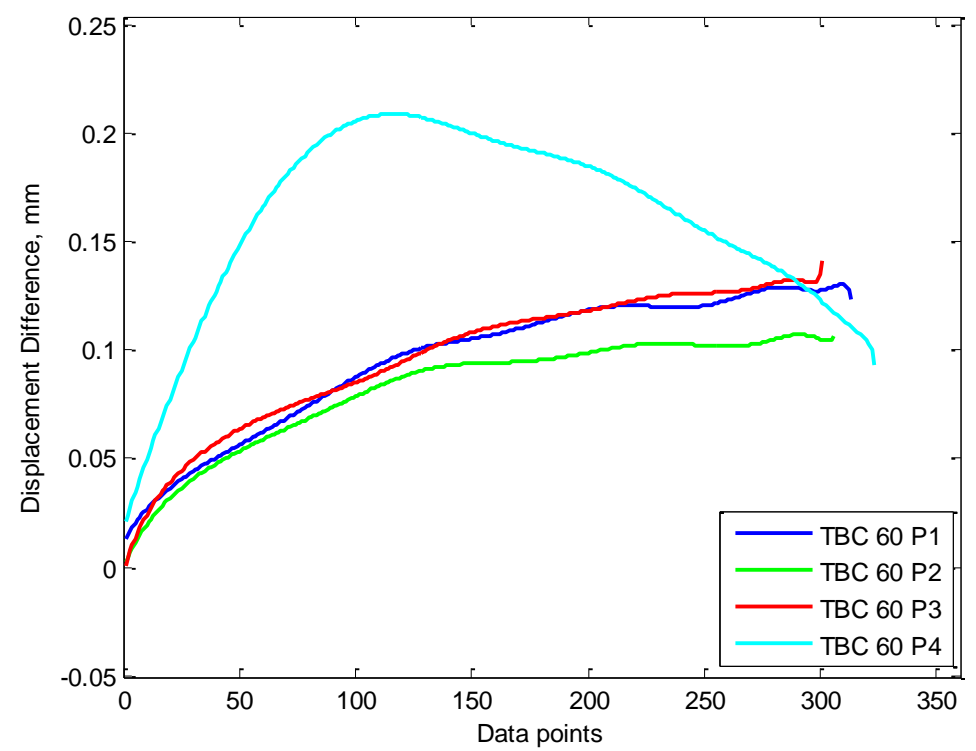

Figure 7. Calculated Displacement Difference for all $\mathbf{T B C} 60^{\circ}$ tests. The difference is taken the same way as for the $30^{\circ}$ differences seen above in Fig. 6 . Only one $60^{\circ}$ panel, P4, was tested in the initial round.

After the correlations between the DIC and DCDT data were calculated for the second round panel data, they were averaged across of the $30^{\circ}$ and $60^{\circ}$ data sets. Once the averaged correlation was applied to each of the initial round DIC data regressions, the inferred DCDT data sets could be generated. Figures 8 and 9 show the final DCDT load-displacement data for each of the $30^{\circ}$ and $60^{\circ}$ panels, respectively. 


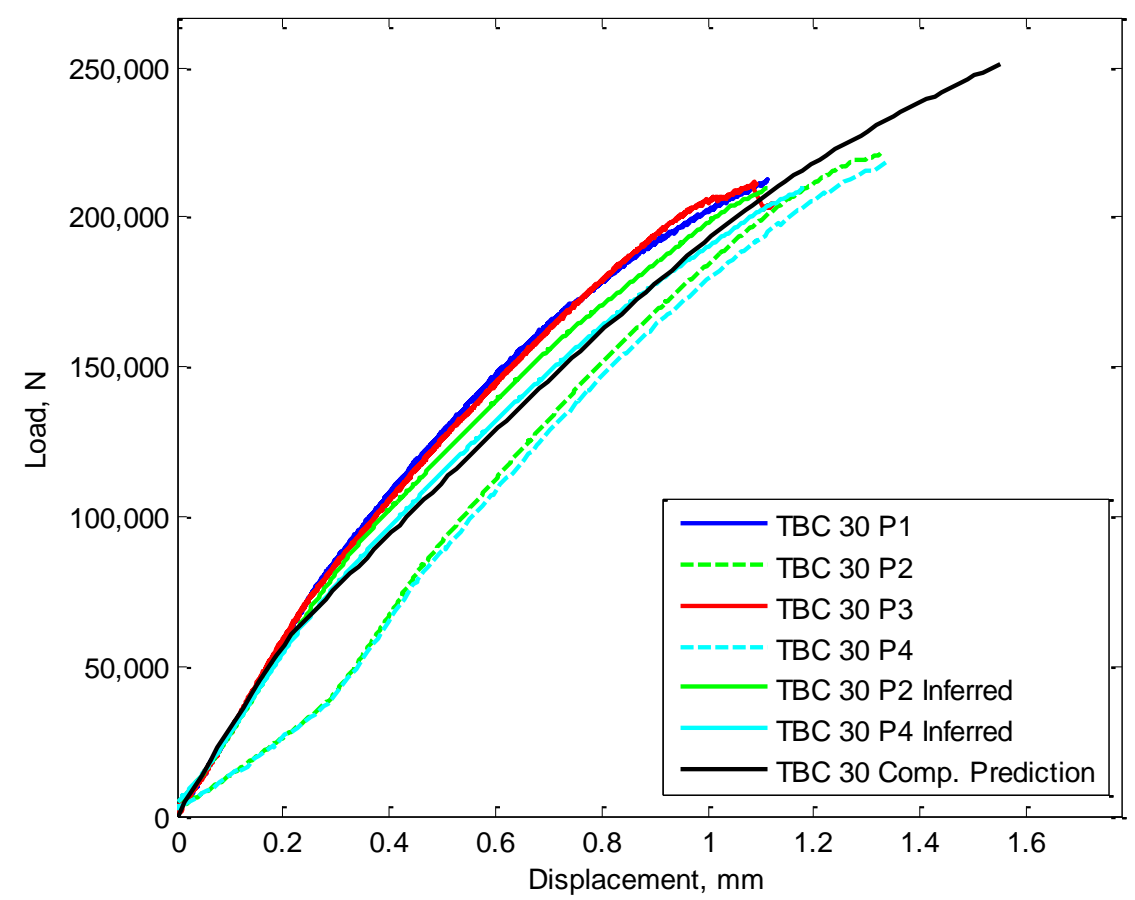

Figure 8. DCDT Data Load-Displacement Curves for $\mathbf{T B C} 30^{\circ}$ Panels. Note the dashed lines are the original DCDT datasets and the inferred data lie between the second round data and the computational prediction.

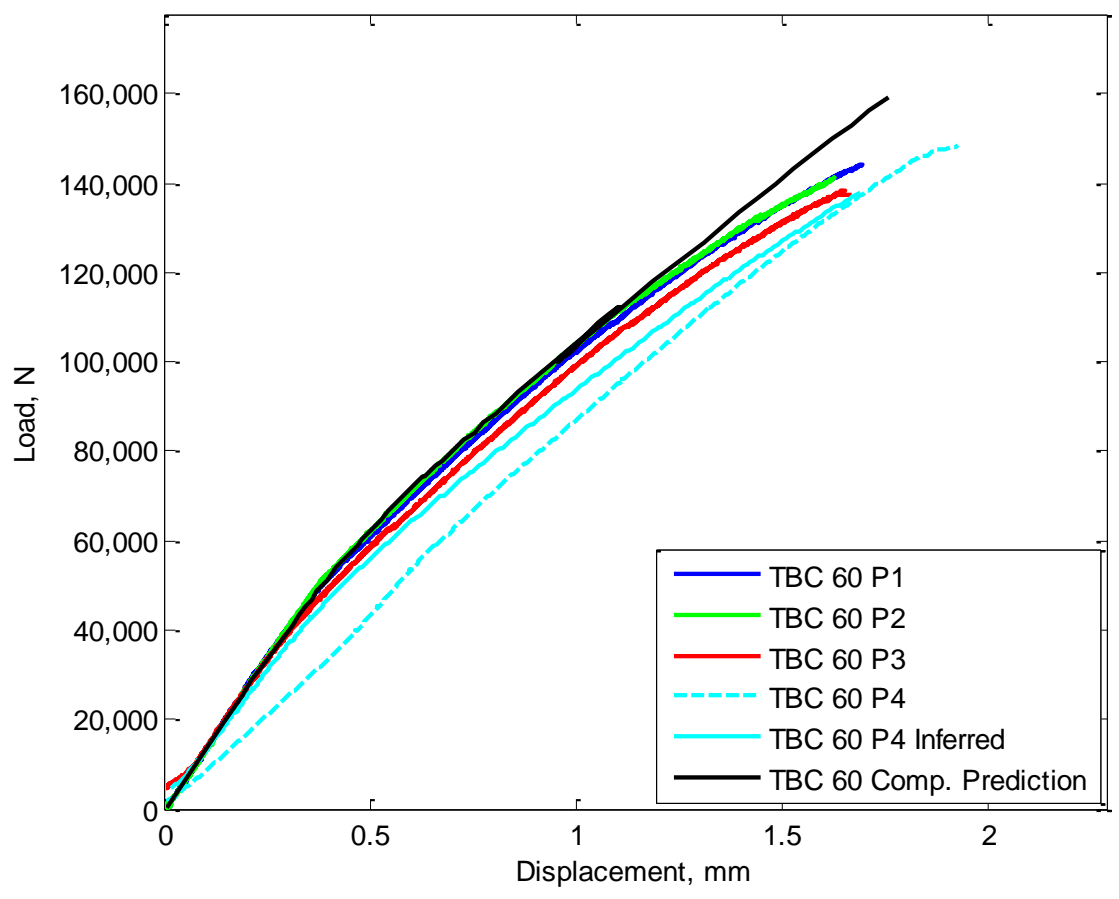

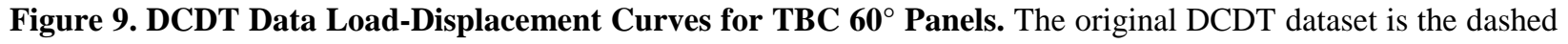
line same as in Fig. 8, but this time the inferred dataset is still the outlier. However, the initial stiffness agreement and overall behavior is much improved over the original dataset. 


\section{Computational Modeling}

A finite element model was developed in the commercial code Abaqus and discussed in detail in Ref. 4. The aim of the model was to correctly capture the initial prebuckling stiffness, buckling load, and initial postbuckling behavior. Deep postbuckling behavior accuracy is the target for future work incorporating nonlinear material modeling and other damaging techniques. Full details of the computational model are discussed in Ref. 4, and Table 1 provides the linear buckling analysis results for reference.

\begin{tabular}{c|cc}
\multicolumn{1}{c}{ Buckling Mode } & $\begin{array}{c}\text { Normalized TBC 30 } \\
\text { Model Load }\end{array}$ & $\begin{array}{c}\text { Normalized TBC 60 } \\
\text { Model Load }\end{array}$ \\
\hline Mode 1 & $49,855 \mathrm{~N} \equiv 1.000$ & $34,893 \mathrm{~N} \equiv 1.000$ \\
Mode 2 & 1.290 & 1.269 \\
Mode 3 & 1.523 & 1.627 \\
Mode 4 & 1.630 & 1.635 \\
Mode 5 & 1.805 & 2.048 \\
\hline
\end{tabular}

Table 1. Normalized Buckling Loads. Obtained by solving a linear buckling analysis (eigenvalue problem).

A general, static step with nonlinear geometry seeded with initial global imperfections from the linear buckling analysis is used to obtain the nonlinear response for both the $30^{\circ}$ and $60^{\circ}$ models. $10 \%$ skin thickness of mode 1 was seeded into the model for the TBC 30 panel, while 10\% skin thickness of mode 1 and $1 \%$ skin thickness of mode 2 was seeded into the model for the TBC 60 panel. The TBC 60 model was seeded with more than mode 1 imperfection due to an observed mode switch behavior during the initial experiments ${ }^{8}$. For certain ratios of mode 1 to mode 2 imperfections, a static Riks arc-length method was used to capture the mode switch behavior ${ }^{7}$ and converge beyond the switching event.

\section{A. Experimental \& Computational Comparison}

The prebuckling stiffnesses for both the TBC $30^{\circ}$ and $60^{\circ}$ models agree well with the experimental data as seen in Figs. 8 and 9. The buckling event was also predicted well as each experiment buckled within a few thousand Newtons of the first mode value in Table 1. The initial postbuckling stiffnesses of the TBC 60 model match well into the postbuckled regime. The TBC 30 model, however, deviates in the initial postbuckling stiffness almost immediately. Figure 10 shows a possible explanation for this behavior through looking at local buckling behavior through the DIC data. Using three extensometer locations along the flat panel face, it becomes clear that the panel does not buckle the same way across the flat panel portions. The wide section suffers the largest loss in stiffness during continued postbuckling loading and closely resembles the computational prediction initial postbuckling stiffness, while the narrow section stiffness appears unchanged deeper into postbuckling until approximately $80 \mathrm{kN}$. The DCDT load-displacement curves shown in Fig. 8 are an average of the four DCDTs used in the test and therefore do not capture this apparent localized behavior. This is an area of current investigation to determine if the boundary conditions need to be reexamined as purely rigid end loading conditions may not be representative of the experimental setup.

The failure load ranged from $210 \mathrm{kN}$ to $220 \mathrm{kN}$ for the TBC $30^{\circ}$ experiments. This is compared to a predicted ${ }^{4}$ failure load of $251 \mathrm{kN}$. This prediction does not include any material nonlinearity or damaging models and is reasonably close to the experimental values at approximately $15 \%$ higher load. The failure load for the $\mathrm{TBC} 60^{\circ}$ experiments ranged from $138 \mathrm{kN}$ to $149 \mathrm{kN}$ compared to the predicted ${ }^{4}$ failure load of $160 \mathrm{kN}$. The prediction is close to the experimental values at approximately $10 \%$ higher without any material nonlinear behavior. Inclusion of nonlinear behavior and/or material damaging into the modeling is a focus for future work and may enhance the accuracy of the predicted failure loads. 


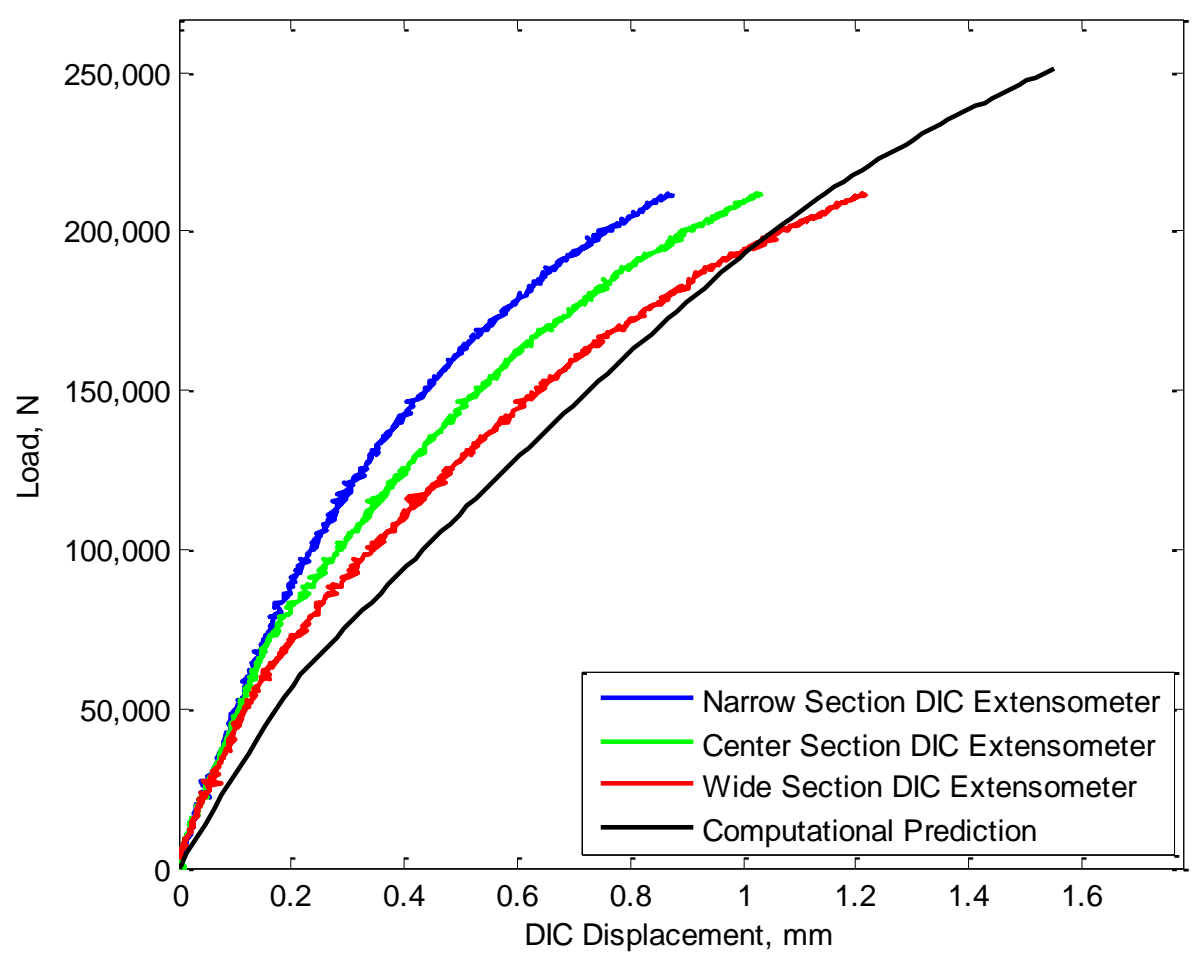

Figure 10. TBC 30 P1 Load-DIC Displacement Curves. Three DIC extensometers were created from the DIC data to show the variations in postbuckling behavior for each of the three flat plate sections. The narrow section is $66 \mathrm{~mm}$ wide, center section is $126 \mathrm{~mm}$ wide, and the wide section is $88 \mathrm{~mm}$ wide as shown in Fig. 2. Note that the DIC extensometer data are generally stiffer than the DCDT instrumentation data. This is not an issue as the DIC data are used for reference only and serve as a backup in the case of the data recovery process.

\section{B. Failure Mode}

A primary goal with the unitized, stiffened panel manufacturing approach was to remove stiffener delamination and debonding as a structural failure mechanism. Not only would removing this mechanism force a different type of failure potentially requiring higher energy, but it would remove the necessity to model and characterize stiffener delamination behavior completely. Figure 11 shows an example of processed DIC data highlighting the presence of cracks after failure of a TBC $30^{\circ}$ panel. Note the formation and propagation of a crack through both the stiffeners and all three sections of the flat plate. The observed failure mechanisms are fiber and matrix cracking due to significant bending and compression loading. The development of the crack in the flat plate sections occurred at essentially the same time as the crack in the stiffeners, and there was no sign of stiffener delamination upon inspection post-testing. The DIC and post-test evaluation indicate that the unitized, stiffened panel concept is capable of successfully removing stiffener delamination as a primary structural failure mode. 

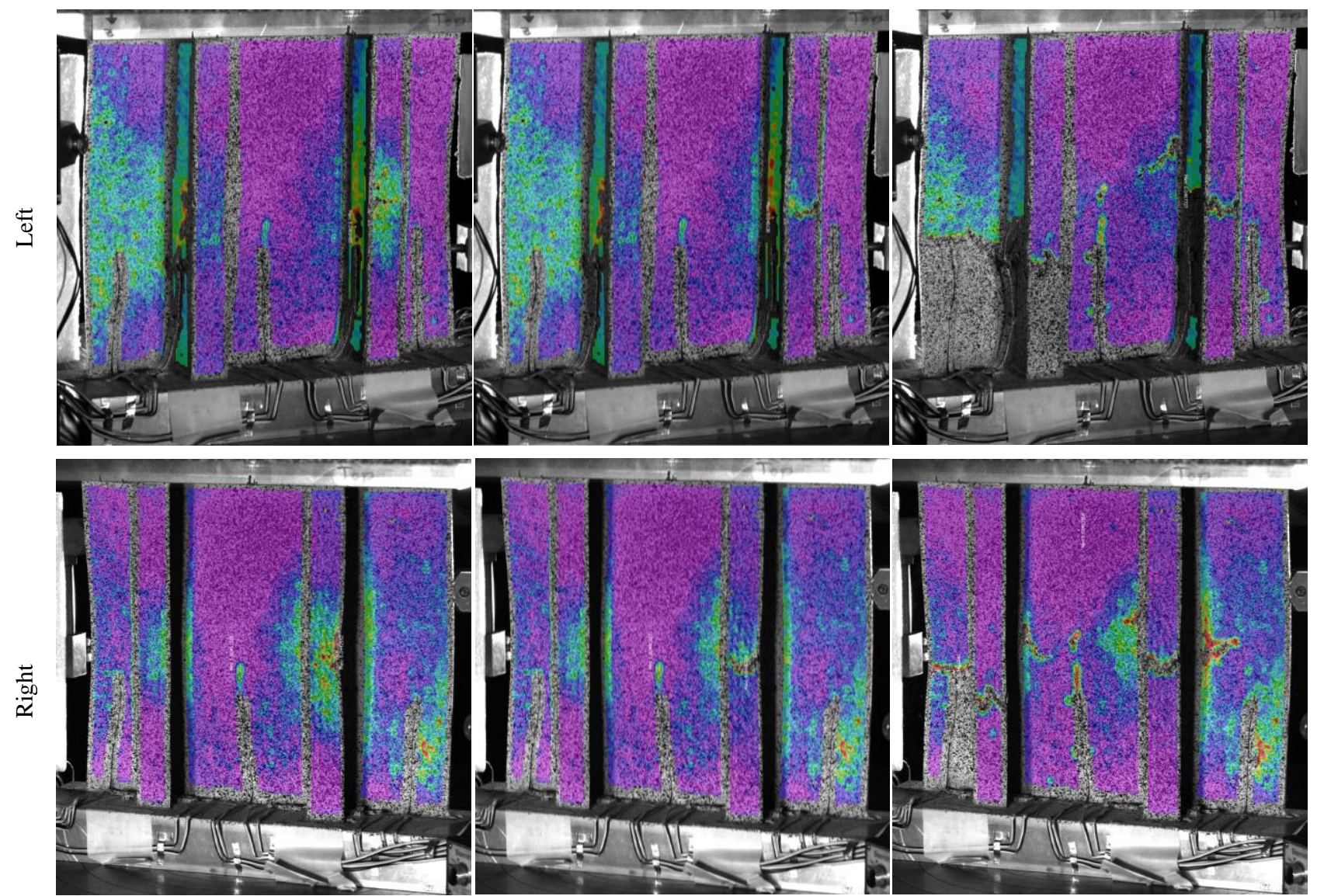

Stiffener Side

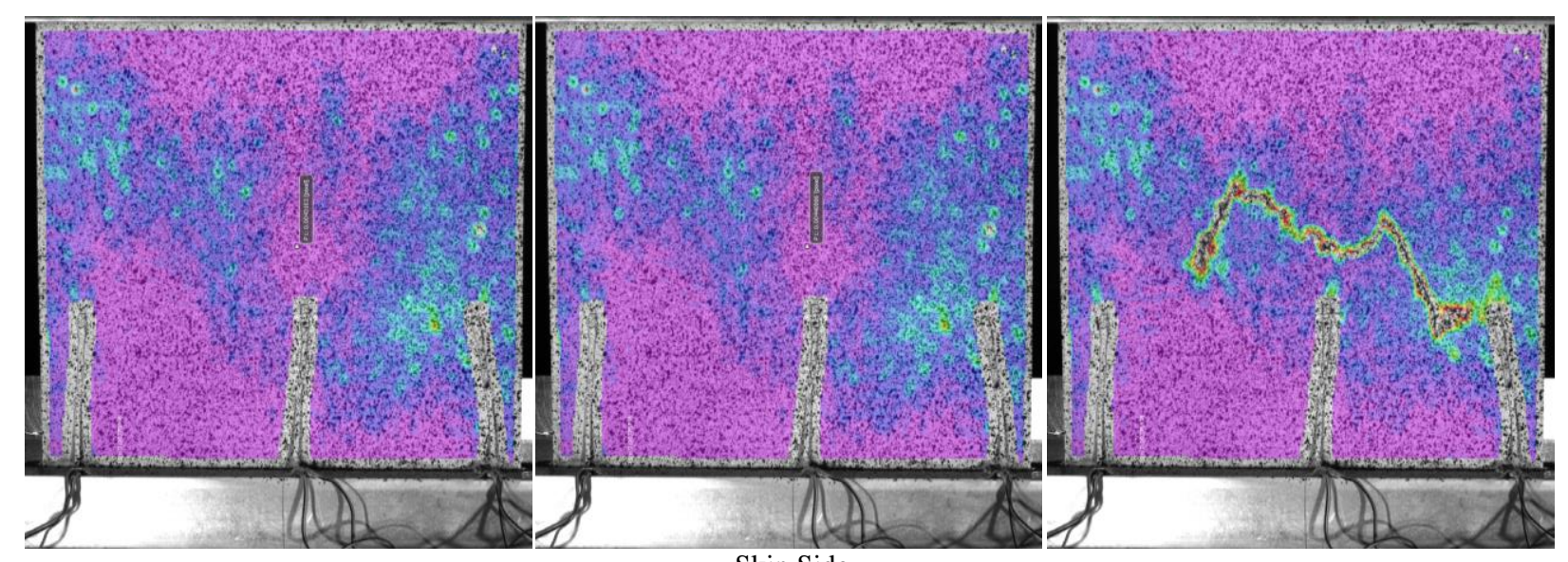

Skin Side

Figure 11. DIC SIGMA Pixels Highlighting Cracks on Failed TBC 30 Specimen. The first two photos in each set of three show the images prior to the failure event, and the third shows the panel just after the failure event. Note the cracking through the stiffeners as well as the flat plate. Similar cracking behavior was observed for TBC 60 specimens as well. 


\section{Conclusion}

This investigation examines the experimental buckling and postbuckling response of unitized, stiffened textile fiber reinforced composite plates. Two dimensional, tri-axially braided textile with two different bias tow angle braids are used for the panels. A method to recover faulty displacement data was provided and demonstrated through recovering three experimental sets of data. This method uses redundant and independent data collection systems (digital image correlation and physical instrumentation) to create a relationship between trusted datasets that may then be applied to recover the faulty data. A computational study of the 2DTBC panels, done previously, is used to compare not only the new experiments, but also the feasibility of the data recovery method. Post-test evaluations indicate the unitized, stiffened panel concept is capable of successfully removing stiffener delamination as a primary structural failure mode. A characterization of the VARTM manufacturing process through fiber volume fraction calculation, initial manufactured geometrical imperfections, and material nonlinear characteristics is significant to capture correct postbuckled behavior, and use of nonlinear material models, non-rigid boundary conditions, and/or failure models may increase the agreement between experimentally observed and computationally predicted postbuckled stiffnesses and failure events.

\section{Acknowledgments}

C. J. R. Kosztowny thanks the NASA Office of the Chief Technologist for funding this work through the Space Technology Research Fellowship, and thanks the NASA Langley Research Center for providing the experimental capabilities used to obtain the provided results. C. J. R. Kosztowny also thanks Dr. Marc R. Schultz for his continued interest, support, and involvement in this project.

\section{References}

${ }^{1}$ Jones, R. M. 1998. Mechanics of Composite Materials. CRC Press, pp. 336-344.

${ }^{2}$ Bisagni, C. 2006. "Progressive Delamination Analysis of Stiffened Composite Panels in Post-Buckling," presented at the $47^{\text {th }}$ AIAA/ASME/ASCE/AHS/ASC Structures, Structural Dynamics, and Materials Conference, May 1-4, 2006.

${ }^{3}$ Jegley, D. C. 2009. "Experimental Behavior of Fatigued Single Stiffener PRSEUS Specimens," NASA Langley Research Center, TM - $2009-215955$.

${ }^{4}$ Kosztowny, C. J. R., and A. M. Waas, 2014. "Buckling and Post-Buckling Behavior of Unitized, Stiffened Tri-Axially Braided Composite Textile Plates," presented at SciTech2014/55 ${ }^{\text {th }}$ AIAA/ASME/ASCE/AHS/ASC Structures, Structural Dynamics, and Materials Conference, January 13-17, 2014.

${ }^{5}$ Quek, S. C., A. M. Waas, K. W. Shahwan, and V. Agaram. 2003. "Analysis of 2D triaxial flat braided textile composites," International Journal of Mechanical Sciences, 45: 1077-1096.

${ }^{6}$ Kier, Z. T., A. Salvi, G. Theis, A. M. Waas, and K. Shahwan. 2010. "Predicting Mechanical Properties of 2D Triaxially Braided Textile Composites Based on Microstructure Properties," Department of Aerospace Engineering, University of Michigan, Ann Arbor.

${ }^{7}$ Brush, D. O., and B. O. Almroth. 1975. Buckling of Bars, Plates, and Shells. McGraw-Hill, Inc, pp. 113-116.

${ }^{8}$ Stoll, F. 1994. "Analysis of the Snap Phenomenon in Buckled Plates," International Journal of Non-Linear Mechanics, Vol. 29, No. 2: $123-138$ 